Editorial

\title{
Vaccination Against Heart Attack?
}

\author{
${ }^{1}$ Joachim Storsberg, ${ }^{2}$ Mark A. Brown and ${ }^{1,3}$ Christian Schmidt \\ ${ }^{1}$ Department of Biomaterials and Healthcare, Fraunhofer Institute for Applied Polymer Research (IAP), \\ Division of Life Science and Bioprocesses, Geiselbergstraße 69, 14476 Potsdam-Golm, Germany \\ ${ }^{2}$ Department of Clinical Sciences and Colorado School of Public Health, \\ Colorado State University, Fort Collins, CO 80523-1052, USA \\ ${ }^{3}$ Editorial Office, The American Journal of Immunology, S-207, 244, 5th Avenue, New York, \\ NY, 10001 USA and S-71, 1A, 400, King William St, Adelaide, SA 5000, Australia
}

Article history

Received: 15-08-2016

Revised: 22-08-2016

Accepted: 22-08-2016

Corresponding Author:

Christian Schmidt

Department of Biomaterials and

Healthcare, Fraunhofer Institute

for Applied Polymer Research

(IAP), Division of Life Science

and Bioprocesses,

Geiselbergstraße 69, 14476

Potsdam-Golm, Germany

Email: christian.schmidt@iap.fraunhofer.de

In the United States, it is estimated that heart attacks strike one individual every $43 \mathrm{sec}$ (Mozaffarian et al., 2016). Correspondingly, $24 \%$ of the recorded deaths in the United States in 2012 were caused by heart disease (Siegel et al., 2016). An analysis of the death certificates for the year 2013 revealed that heart failure was indicated in 1 out of 9 cases (Mozaffarian et al., 2016). All these numbers make one wonder how useful it would be if vaccination could provide protection against heart attacks.

To answer this question, or to provide argument in favor of a systematic clinical trial, one is forced to consult the published record.

In an editorial, Søgaard (2015) alerts readers to the caveat, as pointed out by Ren et al. (2015) as well, that the pneumococcal polysaccharide vaccination was, in some cases, administered to patients with a higher risk of acute coronary syndrome and/or stroke, which would introduce a bias. Furthermore, Søgaard (2015) points out that two studies, namely (Lamontagne et al., 2008; Eurich et al., 2012), report an unusually striking effect of pneumococcal polysaccharide vaccination on acute coronary syndrome when compared to the other studies, evaluated by (Ren et al., 2015).

There is precedent for the use of attenuated or subunit vaccines in the clinical management of infectious diseases. Refer to Borba et al. (2015; Salk and Salk,
1977; Pulendran and Ahmed, 2011) as guide for further reading. This approach hinges on the ability of antibodies to recognize the infectious antigen and mark them for destruction. Logically, one may now ask whether myocardial infarction is an infectious disease in fulfillment of the Henle-Koch postulates (Evans, 1976). There appears to be no indication to the knowledge of the authors that would provide reasonable evidence in support of an answer in the affirmative.

In the apparent absence of direct evidence, one is then forced to consider indirect evidence for either myocardial infarction being an infectious disease in the classical sense or whether there is precedent in the literature that would provide justification above and beyond a reasonable doubt for patient's benefits derived from either an appropriate vaccination event or antibodies generated as a result of an infection. As a result, the lingering argument of whether a true comparison of study population is justified needs careful consideration; we have made similar lines of reasoning available elsewhere and shall incorporate them as stated in appropriate context by reference of (Schmidt and Brown, 2015a; 2015b).

At first glance, there appears to be a serological correlation with higher class $\gamma$ and $\alpha$ antibodies of patients as measured by ELISA and tabulated as highest dilution where an optical density of 0.3 was 
observed (Saikku et al., 1988). The authors reported dilutions of $\geq 128$ for class $\gamma$ and $\geq 32$ for class $\alpha$ antibodies against the Taiwan acute respiratory agent strain of Chlamydia pneumonia (referred to as type strain TW-183 and isolated in 1965 from a child in Taiwan; Grayston et al., 1989; Kuo et al., 1995) with a $p=0.00001$ for acute myocardial infarction group versus control and $\mathrm{p}=0.003$ for chronic coronary heart disease versus control. The authors could not find a significant correlation (defined as $\mathrm{p}>0.05$ ) when the chronic coronary heart disease group was compared with the acute myocardial infarction group (Saikku et al., 1988). Additional support for this notion is provided by a completely independent study where the $C$. pneumonia strain IOL 207 was used to test patient's sera to argue that further effort is needed to shed light onto the pathogenesis of coronary heart pathologies (Mendall et al., 1995).

The above-mentioned evidence points to the involvement of two strains of $C$. pneumonia in pathologies involving coronary heart disease, whilst one strain (TW-182) is implicated to be involved in myocardial infarction, whereas another one, IOL-207, albeit indirectly by virtue of using it as a target to test patients' sera, is ascribed to be of concern in the pathologies of coronary heart disease. If so and how and when and how often, to name a few of the questions that come to mind, remain to be worked out individually and collectively using the whole spectrum of technology available to us; Storsberg and Schmidt (2015) as an introduction.

What about the more than 20 isolates referred to in Grayston et al. (1989)? Are all of them identical with regard to their immunological features? Doubt on this notion was cast by Wagels et al. (1994), where patient's antibodies recognize a pattern of bands in a Western blot of different isolates (Wagels et al., 1994).

Looking from the other side, namely published data pertaining to myocardial infarction and antibodies, one study, for instance, provides data showing that patients at risk of suffering from acute myocardial infarction, at time of analysis, exhibit an elevated level of class $\gamma$ anti-oxidized low-density lipoprotein while after 30 days of follow-up, the levels of these $\gamma$ decreased while the levels of $\mu$ antioxidized low-density lipoprotein increased (all statistically significant with $\mathrm{p}$ values below 0.01 ; Tsimikas et al., 2003). Whilst there is no doubt that the observation is significant, the immunological concept of baseline or alternatively expressed as natural class $\gamma$ antibodies is not easily defined because memory B cells could secrete $\gamma$ and, equally, B cell clones could secrete $\gamma$ in response to contact with foreign material, such as invading pathogens (Gronwall and Silverman, 2014).

The above provides a convincing impetus for a serological monitoring of a given study group to ensure qualified comparisons between study groups are employed if one sets out to argue, say, that there is 'no evidence of association between Chlamydia pneumoniae IgG seropositivity and risks of future myocardial infarction' (quote taken from Ridker et al., 1999; supportive statements are provided by an independent study, e.g., Nieto et al., 1999).

\section{Conclusion}

Serological information accompanying study reports are needed to avoid a comparison of potentially incompatible study groups. $\mathrm{Qu}$ et al. (2013) as an example of highlighted issues surrounding an accurate and precise detection of infectious particles in a group of patients.

\section{Acknowledgement}

Joachim Storsberg and Christian Schmidt gratefully acknowledge support from the Fraunhofer Lighthouse Project Theranostic Implants. Mark A. Brown received financial support from the Short Memorial Endowment and The National Science Foundation (1060548). The content of this paper is entirely the responsibility of the authors and does not necessarily represent the official views of either The Fraunhofer Society for the advancement of applied research or The National Science Foundation.

\section{Author's Contributions}

Joachim Storsberg and Mark A. Brown: Provided critical input and assisted in revising and improving the paper.

Christian Schmidt: Wrote the first draft of the paper and provided critical input and assisted in revising and improving the paper.

\section{Ethics}

Joachim Storsberg, Christian Schmidt and Mark A. Brown report no conflicts of interest with regard to this report. Mark A. Brown and Christian Schmidt are members of the Editorial Board of The American Journal of Immunology, are waived from the Article Processing fee for this contribution and receive no remuneration for the editorial work, either individually or collectively.

\section{References}

Borba, R.C., V.M. Vidal and L.O. Moreira, 2015. The re-emergency and persistence of vaccine preventable diseases. Anais Acad. Bras Cienc., 87: 1311-1322. DOI: 10.1590/0001-3765201520140663 
Eurich, D.T., J.J. Johnstone, J.K. Minhas-Sandhu, T.J. Marrie and S.R. Majumdar, 2012. Pneumococcal vaccination and risk of acute coronary syndromes in patients with pneumonia: Population-based cohort study. Heart, 98: 1072-1077.

DOI: 10.1136/heartjnl-2012-301743

Evans, A.S., 1976. Causation and disease: The HenleKoch postulates revisited. Yale J. Biol. Med., 49: 175-195. PMID: 782050

Grayston, J.T., C.C. Kuo, L.A. Campbell and S.P. Wang, 1989. Chlamydia pneumoniae sp. nov. for Chlamydia sp. strain TWAR. Int. J. Syst. Evol. Microbiol., 39: 88-90. DOI: 10.1099/00207713-39-1-88

Gronwall, C. and G.J. Silverman, 2014. Natural IgM: Beneficial autoantibodies for the control of inflammatory and autoimmune disease. J. Clin. Immunol., 34: S12-S21.

DOI: $10.1007 / \mathrm{s} 10875-014-0025-4$

Kuo, C.C., L.A. Jackson, L.A. Campbell and J.T. Grayston, 1995. Chlamydia pneumoniae (TWAR). Clin. Microbiol. Rev., 8: 451-461. PMID: 8665464

Lamontagne, F., M.P. Garant, J.C. Carvalho, L. Lanthier and M. Smieja et al., 2008. Pneumococcal vaccination and risk of myocardial infarction. CMAJ, 179: 773-777. DOI: 10.1503/cmaj.070221

Mendall, M.A., D. Carrington, D. Strachan, P. Patel and N. Molineaux et al., 1995. Chlamydia pneumoniae: risk factors for seropositivity and association with coronary heart disease. J Infect, 30: 121-128.

Mozaffarian, D., E.J. Benjamin, A.S. Go, D.K. Arnett and M.J. Blaha et al., 2016. Executive Summary: Heart Disease and Stroke Statistics-2016 Update: A Report from the American Heart Association. Circulation, 133: 447-454. DOI: $10.1161 /$ CIR.0000000000000366

Nieto, F.J., A.R. Folsom, P.D. Sorlie, J.T. Grayston and S.P. Wang et al., 1999. Chlamydia pneumoniae infection and incident coronary heart disease: The atherosclerosis risk in communities study. Am. J. Epidemiol., 150: 149-156.

Pulendran, B. and R. Ahmed, 2011. Immunological mechanisms of vaccination. Nat. Immunol., 12: 509-517. DOI: 10.1038/ni.2039

Qu, J., L. Gu, J. Wu, J. Dong, Z. Pu and Y. Gao et al., 2013. Accuracy of IgM antibody testing, FQ-PCR and culture in laboratory diagnosis of acute infection by Mycoplasma pneumoniae in adults and adolescents with community-acquired pneumonia. BMC Infect. Dis., 13: 172-172.

DOI: $10.1186 / 1471-2334-13-172$
Ren, S., D. Newby, S.C. Li, E. Walkom and P. Miller et al., 2015. Effect of the adult pneumococcal polysaccharide vaccine on cardiovascular disease: A systematic review and meta-analysis. Open Heart, 2: e000247-e000247.

DOI: 10.1136/openhrt-2015-000247

Ridker, P.M., R.B. Kundsin, M.J. Stampfer, S. Poulin and C.H. Hennekens, 1999. Prospective study of Chlamydia pneumoniae IgG seropositivity and risks of future myocardial infarction.Circulation, 99: 1161-1164. DOI: 10.1161/01.CIR.99.9.1161

Saikku, P., M. Leinonen, K. Mattila, M.R. Ekman and M.S. Nieminen et al., 1988. Serological evidence of an association of a novel Chlamydia, TWAR, with chronic coronary heart disease and acute myocardial infarction. Lancet, 2: 983-986.

DOI: 10.1016/S0140-6736(88)90741-6

Salk, J. and D. Salk, 1977. Control of influenza and poliomyelitis with killed virus vaccines. Science, 195: 834-847. DOI: 10.1126/science. 320661

Schmidt, C. and M.A. Brown, 2015a. Relating the pendulum of democracy with oncology research. J. Clin. Exp. Oncol., 4: 1-3. DOI: $10.4172 / 2324-9110.1000 \mathrm{e} 110$

Schmidt, C. and M.A. Brown, 2015b. The sins of omission. J. Clin. Exp. Oncol. DOI: $10.4172 / 2324-9110.1000 \mathrm{e} 109$

Siegel, R.L., K.D. Miller and A. Jemal, 2016. Cancer statistics, 2016. CA: Cancer J. Clin., 66: 7-30. DOI: $10.3322 /$ caac. 21332

Søgaard, O.S., 2015. Does pneumococcal vaccination prevent myocardial infarction and stroke in elderly adults? Open Heart, 2: e000306-e000306. DOI: 10.1136/openhrt-2015-000306

Storsberg, J. and C. Schmidt, 2015. Nanomaterialstools, technology and methodology of nanotechnology based biomedical systems for diagnostics and therapy. Biomedicines, 3: 203-223. DOI: 10.3390/biomedicines3030203

Tsimikas, S., C. Bergmark, R.W. Beyer, R. Patel and J. Pattison et al., 2003. Temporal increases in plasma markers of oxidized low-density lipoprotein strongly reflect the presence of acute coronary syndromes. J. Am. Coll. Cardiol., 41: 360-370. DOI: $10.1016 / \mathrm{S} 0735-1097(02) 02769-9$

Wagels, G., S. Rasmussen and P. Timms, 1994. Comparison of Chlamydia pneumoniae isolates by western blot (immunoblot) analysis and DNA sequencing of the omp 2 gene. J. Clin. Microbiol., 32: 2820-2823. PMID: 7852577 